\title{
Cortisol production during high dose dexamethasone therapy in neurological and neurosurgical patients
}

\author{
TRO' R BROPHY, JB CHALK, K RIDGEWAY, JH TYRER, MJ EADIE \\ From the University Department of Surgery and Medicine, Royal Brisbane Hospital, Brisbane, Australia
}

SUMMARY Simultaneous plasma dexamethasone and cortisol levels were followed at intervals over 8 hour periods on 40 occasions in 19 subjects who received regular high dosage dexamethasone therapy (rarely less than $12 \mathrm{mg}$ a day) for various neurological and neurosurgical conditions. Lower dexamethasone doses (for example $2 \mathrm{mg}$ daily for 2 days) normally suppress adrenal cortical production of cortisol to below $50 \mu \mathrm{g} / \mathrm{l}$ for at least 8 hours. However, in 12 of the 35 studies that did not take place at the first steroid dose or in subjects taking second daily bolus steroid dosage such suppression was not present 8 to 12 hours after dexamethasone intake, though it was shown that dexamethasone could suppress cortisol production in all these cases. Failure of maintained suppression despite the high steroid dose appeared to be related to rapid elimination of dexamethasone. These findings may help explain the relative rarity of adrenal failure in clinical neurological practice after high dosage steroid therapy is ceased.

Oral dexamethasone intake is sometimes used to suppress adrenal production of cortisol, as a test of control of the pituitary-adrenal axis. The administered glucocorticoid should act on the hypothalamus and pituitary to inhibit corticotropin formation, and this in turn should remove the stimulus for adrenal production of cortisol. The usual oral dexamethasone dose is 0.5 or $1.0 \mathrm{mg}$, given every 6 hours for 48 hours. The criterion of adrenal suppression is taken as a morning peak plasma cortisol level below $50 \mu \mathrm{g} / \mathrm{l}(138 \mathrm{nmol} / \mathrm{l})$ present 8 hours following the most recent dexamethasone dose. A variant of this test has come into use in contemporary psychiatry, as an aid in the diagnosis of depression.' A single dexamethasone oral dose of $1 \mathrm{mg}$ in non-depressed normal persons should suppress plasma cortisol levels to below 40 or $50 \mu \mathrm{g} / \mathrm{l}$ for at least 8 hours, and often for 24 hours: suppression to such a level may not occur in depressed persons.

In neurological and neurosurgical practice high oral doses of dexamethasone (for example 4 to $8 \mathrm{mg}$ of the base 6 or 8 hourly) are sometimes used for days or weeks for a variety of purposes such as to

Address for reprint requests: Prof Mervyn Eadie, Dept of Medicine, Clinical Sciences Building, Royal Brisbane Hospital, Brisbane 4029, Australia

Received 4 January 1984 and in revised form 29 March 1984. Accepted 1 April 1984 retard the progression of cerebral tumours and diminishing oedema in and around such tumours. Bearing in mind the size of these steroid doses, the frequency of drug administration and the known suppression of adrenal cortisol production by the substantially lower glucocorticoid doses used in dexamethasone suppression tests, one would anticipate profound and continuous suppression of endogenous cortisol production in patients receiving high dose dexamethasone therapy several times a day for neurological and neurosurgical disorders.

During various studies of the pharmacokinetics of dexamethasone in neurological and neurosurgical patients we have carried out simultaneous assays of endogenous plasma cortisol levels, intending to gain insight into the severity of the adrenal cortical suppression produced. The degree of suppression has not always proved as great as expected. The findings are here reported as they help explain the relative infrequency of adrenal cortical insufficiency manifestations after high dose steroid therapy is withdrawn in neurological patients.

\section{Materials and methods}

During a series of investigations of dexamethasone pharmacokinetics, simultaneous plasma cortisol levels were measured at intervals after dexamethasone dosage in 40 studies carried out in 19 
Table 1 Details of the subjects studied

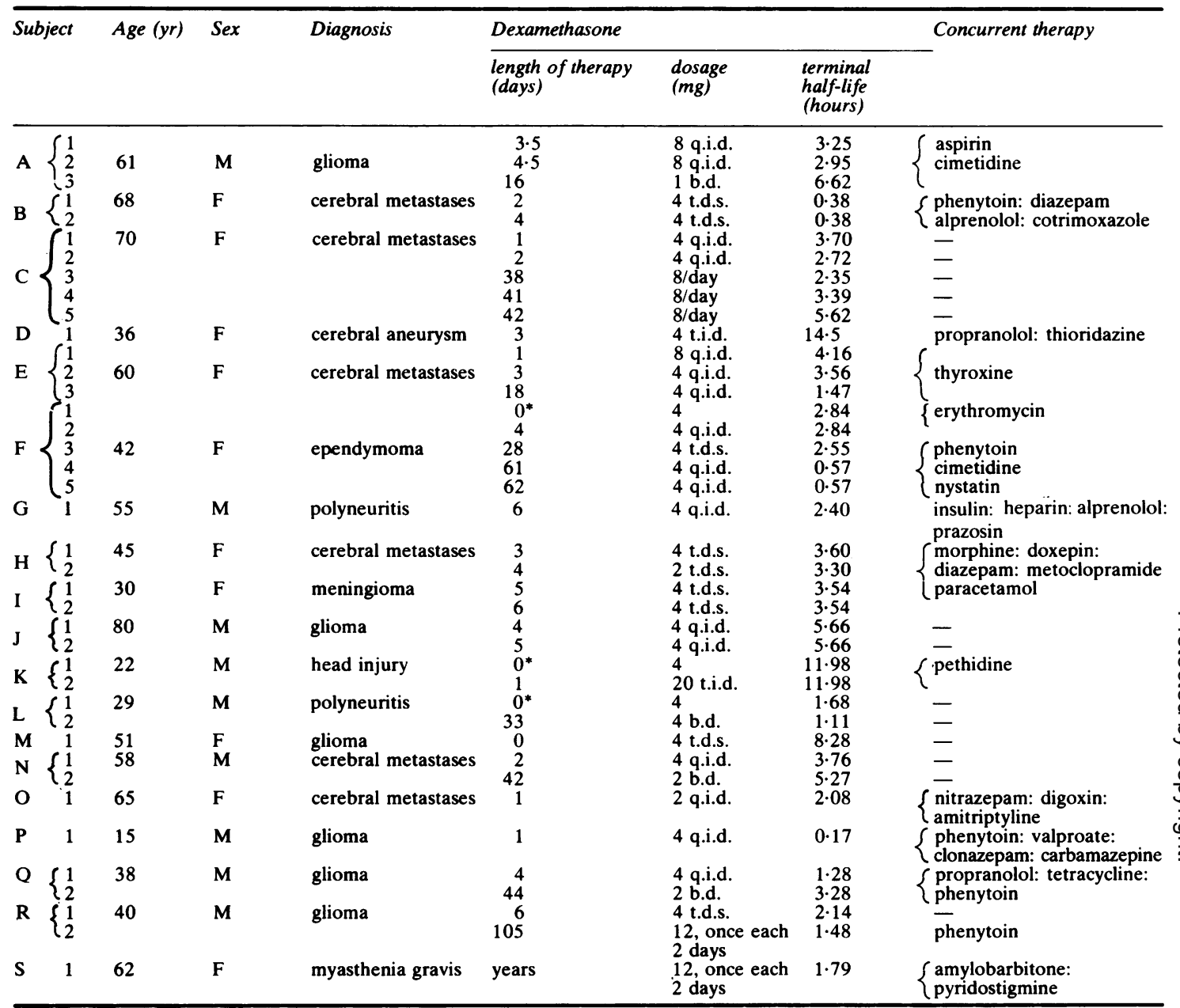

* = first dexamethasone dose

neurological or neurosurgical patients. Personal details of the patients studied, of their diagnoses, durations of dexamethasone therapy prior to each study in which they were involved, and details of their dexamethasone dosages are provided in table 1 , together with their dexamethasone terminal plasma half life values at the time of study, and details of any concurrent therapy.

These dexamethasone pharmacokinetic studies were carried out in volunteers who gave informed consent during the course of high dosage steroid therapy given for a variety of neurological or neurosurgical indications. The investigational protocol had received institutional ethics committee approval. Single oral or intravenous dexamethasone doses, nearly always equivalent to $4 \mathrm{mg}$ of the base, were given, on almost all occasions between 8 and $10 \mathrm{am}$. The most recent dexamethasone dose had been taken 8-12 hours before, in nearly all instances. Forearm venous blood collections were carried out as near as possible to the following times in relation to the test dose of dexamethasone: 0 , $0 \cdot 16,0 \cdot 33,0 \cdot 50,0 \cdot 75,1 \cdot 0,1 \cdot 5,2 \cdot 0,2 \cdot 5,3 \cdot 0,4 \cdot 0$, $5 \cdot 0,6 \cdot 0$ and $8 \cdot 0$ hours. Plasma dexamethasone and cortisol concentrations were measured in each blood sample, using the assay described below. The plasma dexamethasone concentration-time data were analysed in terms of a two compartment open linear 
pharmacokinetic model with elimination taking place from the central compartment. The concentration-time data were fitted iteratively to the model using an Apple 11 plus microcomputer running either the Basic language least squares curve fitting program described by Peck and Barrett, ${ }^{2}$ derived from the program originally written by Horwitz and Homer, ${ }^{3}$ or a Pascal language iterative curve fitting program also utilising the Marquardt algorithm ${ }^{4}$ and supplied by Stemsoft Pty. Ltd. Halflife values were calculated from the terminal rate constant $(\beta)$ of the plasma dexamethasone level decline in the usual way, viz.

$$
\mathrm{T}_{1 / 2(\beta)}=\frac{\log _{\mathrm{n}} 2}{\beta}
$$

\section{Plasma steroid measurement}

The assay used was a modification of the high performance liquid chromatographic method originally described by Cham et al. ${ }^{5}$ With the modification it was possible to measure simultaneous plasma concentrations of dexamethasone and cortisol in each sample in a single assay run. After addition of $100 \mu \mathrm{l}$ ethanolic cyheptamide $(100 \mu \mathrm{g} / \mathrm{ml})$, to serve as an internal standard, $3 \mathrm{ml}$ plasma samples were made alkaline with $\mathrm{NaOH}$ and extracted with $\mathrm{N}$-heptane. The organic phase was aspirated to waste, and the inorganic phase mixed with $300 \mathrm{mg} \mathrm{NaCl}$ and 100 $\mathrm{ml}$ dichloromethane. The organic phase was then separated off, evaporated to dryness, reconstituted in $50 \mu \mathrm{l}$ methanol and injected into a high performance liquid chromatography system comprising a Waters Associates M6000 A pump, a Bondapak C18 column, and a Waters Associates Model 440 absorbance detector set at a wavelength of $254 \mathrm{~nm}$. The mobile phase used was a mixture of acetic acid 12 parts, butanol 75 parts, methanol 40 parts and water 873 parts, at room temperature and a flow rate of $2.5 \mathrm{ml} /$ minute at a pressure of $23333 \mathrm{kPa}$. With this system retention times were, respectively $7.8,13.4$ and 16.5 minutes for cortisol, dexamethasone and cyheptamide. Quantitation was by the peak height ratio method.

\section{Results}

The typical time-courses of plasma dexamethasone and cortisol concentrations after an intravenous dose of dexamethasone given to a patient are shown in fig 1 . As was nearly always the case, the time course of the cortisol levels lagged 1-2 hours behind the dexamethasone levels. The time-courses of these parameters in subject $F$, when measured on two occasions two months apart, are shown in fig 2 , phenytoin therapy having enhanced the elimination

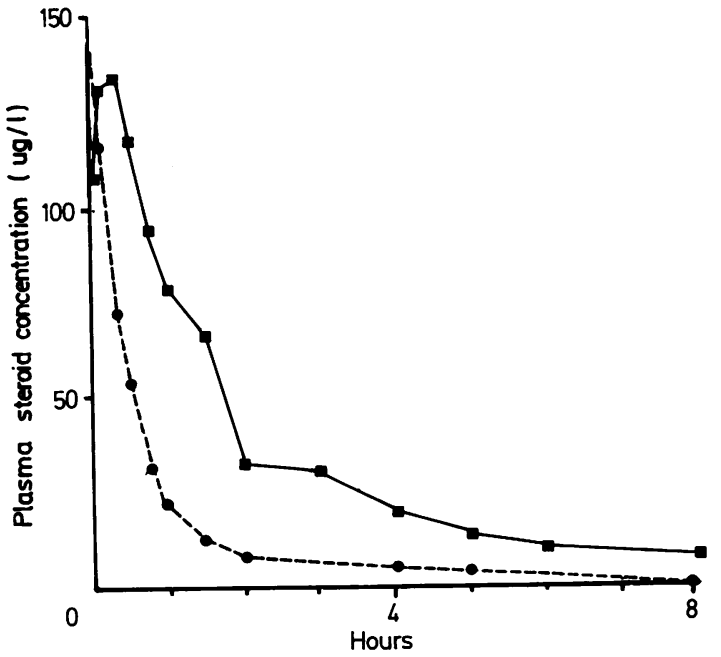

Fig 1 Time course of plasma dexamethasone (broken line) levels after an intravenous $4 \mathrm{mg}$ dose in subject 1 , who had received oral dexamethasone $16 \mathrm{mg}$ per day for 5 days by the time of study. Plasma cortisol levels (continuous line) fell from the lower limits of the physiological range to values below $10 \mu \mathrm{g} / \mathrm{l}$, tending to lag 1-2 hours behind the dexamethasone level.
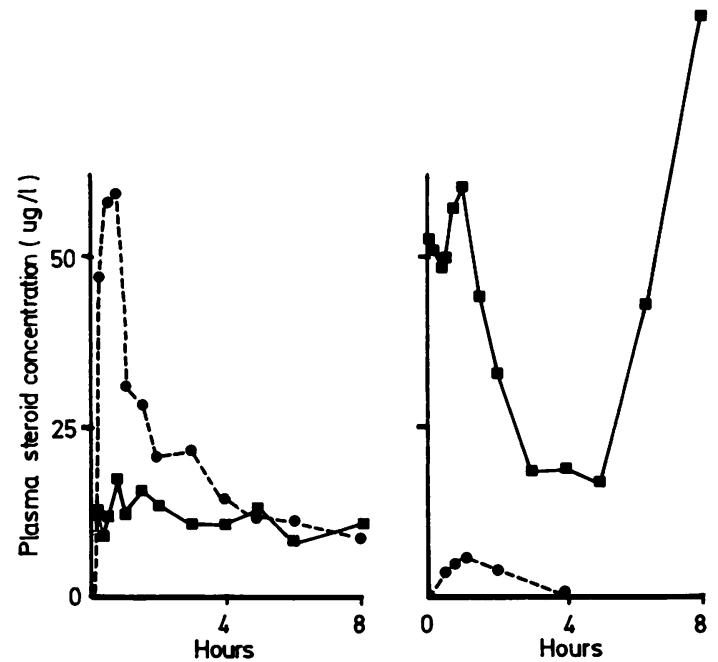

Fig 2 Time courses of plasma dexamethasone (broken line) and plasma cortisol levels (continuous line) following $4 \mathrm{mg}$ oral dexamethasone doses in subject $F$, who had received $16 \mathrm{mg}$ of dexamethasone over 24 hours prior to the first study, and who had taken dexamethasone 12-16 mg/day for 2 months prior to the second study. Phenytoin therapy had been commenced between the two studies, and dexamethasone elimination was much greater by the time of the second study. Plasma cortisol production was suppressed throughout the first study, but was not continuously suppressed at the time of the second study, though it did suppress, briefly. 
Table 2 Plasma dexamethasone and cortisol responses in the subjects studied

\begin{tabular}{|c|c|c|c|c|c|c|c|c|c|}
\hline \multirow[t]{2}{*}{ Subject } & \multicolumn{4}{|c|}{ Dexamethasone $(\mu \mathrm{g} / \mathrm{l})$} & \multicolumn{5}{|c|}{ Cortisol $(\mu g / l)$} \\
\hline & Co & $C \max$ & $T \max (h r)$ & $C(T \min )$ & Co & $C \max$ & $T \max (h r)$ & $C \min$ & $T \min (h r)$ \\
\hline 1 & $5 \cdot 8$ & $67 \cdot 7$ & $0 \cdot 5$ & $5 \cdot 82$ & 14.7 & 14.7 & 0 & $10 \cdot 1$ & $8 \cdot 25^{*}$ \\
\hline$A\} 2$ & 0 & $106 \cdot 2$ & 0.16 & $14 \cdot 0$ & 18.4 & $15 \cdot 4$ & 1 & 9.8 & \\
\hline & $4 \cdot 4$ & $126 \cdot 7$ & $0 \cdot 16$ & $36 \cdot 3$ & $18 \cdot 2$ & $49 \cdot 1$ & $0 \cdot 16$ & 15.4 & \\
\hline 1 & 0 & $58 \cdot 5$ & $0 \cdot 16$ & 0 & $156 \cdot 1$ & $156 \cdot 1$ & 0 & 34.8 & $8^{*}$ \\
\hline 2 & 0 & $13 \cdot 7$ & $0 \cdot 16$ & 0 & 156.7 & 157.6 & $0 \cdot 16$ & $46 \cdot 0$ & $8^{*}$ \\
\hline 1 & 0 & 33.0 & 0.53 & 0 & $17 \cdot 3$ & 20.5 & 1.03 & $10 \cdot 0$ & $8.67^{*}$ \\
\hline 2 & 0 & 88.2 & $0 \cdot 18$ & $12 \cdot 5$ & 10.9 & 10.9 & 0 & $8 \cdot 2$ & $4 \cdot 67$ \\
\hline C & $9 \cdot 5$ & $32 \cdot \overline{5}$ & $1 \cdot 10$ & $21 \cdot 5$ & $11 \cdot 7$ & 11.9 & $0 \cdot 16$ & $5 \cdot 7$ & 4 \\
\hline 4 & 0 & $136 \cdot 6$ & $0 \cdot 16$ & $<10$ & $24 \cdot 1$ & $27 \cdot 4$ & 0.33 & $11 \cdot 2$ & $8^{*}$ \\
\hline 15 & 0 & 133.7 & $0 \cdot 16$ & $15 \cdot 02$ & $21 \cdot 0$ & 24.9 & 0.52 & $16 \cdot 0$ & $4 \cdot 08$ \\
\hline D $\int_{1}^{1}$ & $\begin{array}{c}0 \\
36 \cdot 2\end{array}$ & $118 \cdot 5$ & $0 \cdot 16$ & $36 \cdot 6$ & $14 \cdot 3$ & $37 \cdot 3$ & 0.33 & $15 \cdot 4$ & $1^{*}$ \\
\hline $\mathrm{E}\left\{\begin{array}{l}1 \\
2\end{array}\right.$ & $\begin{array}{c}30 \cdot 2 \\
0\end{array}$ & $\begin{array}{l}46 \cdot 1 \\
96 \cdot 0\end{array}$ & 0.75 & 8.05 & $15 \cdot 2$ & $15 \cdot 2$ & 0 & $8 \cdot 3$ & $8^{*}$ \\
\hline$l_{3}^{2}$ & $26 \cdot 0$ & $\begin{array}{r}96 \cdot 0 \\
254 \cdot 2\end{array}$ & $\begin{array}{l}0 \cdot 16 \\
0 \cdot 16\end{array}$ & $9 \cdot 26$ & $31 \cdot 1$ & $31 \cdot 1$ & 0 & $11 \cdot 0$ & $8 \cdot 33^{*}$ \\
\hline $1 \dagger$ & 0 & 87.2 & 0.16 & $\stackrel{?}{2} \cdot 82$ & $\begin{array}{r}14 \cdot 2 \\
114.5\end{array}$ & $\begin{array}{r}17 \cdot 8 \\
180 \cdot 0\end{array}$ & $0 \cdot 16$ & 9.4 & $7^{*}$ \\
\hline 2 & 0 & $59 . \overline{7}$ & 0.75 & 11.0 & $12 \cdot 3$ & $\begin{array}{r}180 \cdot 0 \\
17 \cdot 5\end{array}$ & $0 \cdot 33$ & $21 \cdot 6$ & $8^{*}$ \\
\hline$F$ & 0 & 111.0 & $0 \cdot 16$ & $5 \cdot 73$ & 14.9 & $15 \cdot 3$ & $\begin{array}{l}0 \cdot 45 \\
0.16\end{array}$ & $8 \cdot 6$ & 6 \\
\hline 4 & 0 & $5 \cdot 2$ & 1 & 0 & 52.0 & 60.5 & $\begin{array}{l}0 \cdot 16 \\
1\end{array}$ & $9 \cdot 5$ & $4 \cdot 58$ \\
\hline 5 & 0 & $68 \cdot 1$ & $0 \cdot 18$ & 0 & 79.0 & 79.0 & $\begin{array}{l}1 \\
0\end{array}$ & $16 \cdot 7$ & 5 \\
\hline 1 & $18 \cdot 7$ & $50 \cdot 0$ & 0.75 & - & 5.6 & $12 \cdot 6$ & $\begin{array}{l}0 \\
0 \cdot 50\end{array}$ & $13 \cdot 4$ & 5 \\
\hline $\mathrm{H}\{1$ & 0 & 122.5 & $0 \cdot 16$ & $12 \cdot 7$ & $23 \cdot 2$ & 37.7 & $\begin{array}{l}0.50 \\
0.38\end{array}$ & $\overline{15 \cdot 7}$ & $\overline{5^{*}}$ \\
\hline 2 & $8 \cdot 3$ & $179 \cdot 2$ & $0 \cdot 16$ & 14.5 & 75.9 & $82 \cdot 4$ & $0 \cdot 16$ & $18 \cdot 1$ & 6 \\
\hline 1 & 0 & $415 \cdot 6$ & $0 \cdot 10$ & 0 & $108 \cdot 9$ & 135.9 & 0.33 & $7 \cdot 3$ & $8^{*}$ \\
\hline 2 & 0 & $12 \cdot 4$ & $1 \cdot 0$ & 0 & $43 \cdot 1$ & $43 \cdot 1$ & 0 & 3.6 & $8^{*}$ \\
\hline 1 & $42 \cdot 9$ & $64 \cdot 5$ & 0.75 & $48 \cdot 3$ & $20 \cdot 7$ & $21 \cdot 7$ & 0.33 & $15 \cdot 5$ & $6^{*}$ \\
\hline 2 & $18 \cdot 6$ & $136 \cdot 3$ & $0 \cdot 16$ & $26 \cdot 8$ & 23.8 & 25.0 & 0.50 & $16 \cdot 5$ & $4^{*}$ \\
\hline 1 & 0 & $207 \cdot 1$ & $1 \cdot 67$ & $111 \cdot 5$ & 53.9 & $143 \cdot 2$ & 0.33 & $18 \cdot 8$ & $8^{*}$ \\
\hline 2 & 0 & 395.6 & $0 \cdot 16$ & $109 \cdot 1$ & 10.4 & $10 \cdot 4$ & 0 & $3 \cdot 5$ & 3 \\
\hline$L\left\{1^{\dagger}\right.$ & 0 & 393.0 & 0.50 & $17 \cdot 2$ & 247.0 & $247 \cdot 0$ & 0 & $14 \cdot 8$ & $7 \cdot 84^{*}$ \\
\hline 12 & $?$ & $285 \cdot 0$ & $0 \cdot 50$ & $40 \cdot 8$ & 85.7 & $85 \cdot 7$ & 0 & 3.9 & $8^{*}$ \\
\hline$M 1$ & 66 & $448 \cdot 0$ & 0.50 & $23 \cdot 3$ & $37 \cdot 5$ & $47 \cdot 9$ & 0.50 & $21 \cdot 0$ & 6 \\
\hline $\mathbf{N}$ & $16 \cdot 8$ & $86 \cdot 1$ & $0 \cdot 25$ & $44 \cdot 5$ & $9 \cdot 0$ & $17 \cdot 4$ & 1.0 & $5 \cdot 0$ & 2 \\
\hline . & $29 \cdot 2$ & 136.4 & $9 \cdot 25$ & $107 \cdot 0$ & $10 \cdot 9$ & 13.6 & 0.5 & $7 \cdot 4$ & 2 \\
\hline O & 0 & $105 \cdot 1$ & $0 \cdot 16$ & 8.96 & $20 \cdot 1$ & $28 \cdot 5$ & 0.5 & $17 \cdot 5$ & $8^{*}$ \\
\hline 1 & 0 & $162 \cdot 3$ & $0 \cdot 16$ & 10 & $151 \cdot 6$ & $204 \cdot 6$ & $0 \cdot 16$ & $34 \cdot 3$ & 3 \\
\hline $0\{1$ & 0 & $61 \cdot 5$ & 0.25 & 0 & 164.7 & 168.4 & 0.5 & $26 \cdot 8$ & 4 \\
\hline 2 & 0 & $83 \cdot 5$ & $0 \cdot 25$ & 6.96 & $154 \cdot 3$ & $154 \cdot 3$ & 0 & $9 \cdot 8$ & $6^{*}$ \\
\hline$R\left\{\begin{array}{l}1 \\
?\end{array}\right.$ & $7 \cdot 69$ & $138 \cdot 17$ & $2 \cdot 00$ & $65 \cdot 73$ & $9 \cdot 5$ & $9 \cdot 5$ & 0 & $2 \cdot 7$ & 6 \\
\hline s 22 & 0 & 35.0 & 1.00 & $3 \cdot 83$ & 68.7 & $68 \cdot 7$ & 1 & $7 \cdot 7$ & 8 \\
\hline $\begin{array}{ll}3 & 1\end{array}$ & 0 & 117.4 & $2 \cdot 08$ & $17 \cdot 5$ & $77 \cdot 4$ & $77 \cdot 4$ & 0 & $9 \cdot 0$ & 7.08 \\
\hline
\end{tabular}

${ }^{*}$ Last sampling time in study

† Studied after first dexamethasone dose

$\mathrm{Co}=$ initial concentration

$\mathrm{Cmax}=$ maximum concentration, occuring at the $\mathrm{T} \max$

$\mathrm{C}(\mathrm{Tmin})=$ plasma concentration of dexamethasone at the time of minimum plasma level of cortisol (Cmin, occurring at Tmin)

of dexamethasone in the interval between the two studies. Table 2 sets out, for each study in each subject, the plasma dexamethasone concentration present at the outset of the study (a residue from the previous dexamethasone dose), the peak plasma dexamethasone level attained in the study and its time of occurrence. This peak value, and the time at which it occurred, varied depending on whether the dexamethasone was given intravenously or orally. Table 2 also shows the plasma cortisol concentration present at the outset of each study, the maximum and the minimum cortisol concentration which developed during each study, and their times of occurrence. The maximum cortisol concentration did not always occur at the outset of a study, as cortisol suppression from a previous dexamethasone dose might still be wearing off at this stage. The plasma dexamethasone concentration coinciding with the time of minimum plasma cortisol level is also shown in Table 2 .

In all 40 studies, the dexamethasone dose given produced a decrease in plasma cortisol levels, from a mean peak value of $63.7 \pm$ SD $64.6 \mu \mathrm{g} / 1$ to a mean minimum value of $13 \cdot 8 \pm 9 \cdot 2 \mu \mathrm{g} / \mathrm{l}$. The true depression in plasma cortisol levels may have been greater than this, since in 20 of the 40 studies the apparent minimum value occurred at the time of the final sampling. In these subjects the true minimum may have occurred later. Apparent minimum cortisol values occurred a mean of $5.50 \pm$ SD 1.99 hours from time of maximum plasma dexamethasone concentrations. In every subject the minimum plasma 
cortisol level in response to dexamethasone was below the $50 \mu \mathrm{g} / \mathrm{l}$ value that is taken as the criterion for suppression of cortisol production.

Either the initial, or the maximum, plasma cortisol concentration was above the $50 \mu \mathrm{g} / \mathrm{l}$ criterion in 15 of the 40 studies. Three of these studies had been carried out at the time of the first dexamethasone dose given to the subject, so that suppressed values would not be expected at this time. However in 12 studies, despite previous and continuing high dose dexamethasone therapy, maximum plasma cortisol concentrations ranged from $60 \cdot 5$ to $204.6 \mu \mathrm{g} / \mathrm{l}$, with six of the maximum values exceeding $100 \mu \mathrm{g} / \mathrm{l}$. When reasons for these 12 apparent failures of suppression of cortisol production from previous dexamethasone dosage were sought the following points emerged:

(1) all but one subject with maximum plasma cortisol levels above $50 \mu \mathrm{g} / \mathrm{l}$ had unmeasurably low plasma dexamethasone levels at the outset of study. The one exception $\left(\mathrm{H}_{2}\right)$ had an initial dexamethasone level of only $8.3 \mu \mathrm{g} / \mathrm{l}$. In contrast there were measurable plasma dexamethasone levels in 12 of the 24 studies in which plasma cortisol levels were below $50 \mu \mathrm{g} / \mathrm{l}$ throughout the study.

(2) two studies (R2 and S) were carried out in subjects receiving a second daily oral bolus dosage regime of $12 \mathrm{mg}$ dexamethasone, and the last dexamethasone dose had been taken 48 hours before the study.

(3) in the remaining 10 studies (B1, B2, F4, F5, $\mathrm{H} 2, \mathrm{I} 1, \mathrm{~L} 2, \mathrm{P} 1, \mathrm{Q} 1, \mathrm{Q} 2)$ mean dexamethasone half life was $1.60 \pm$ SD 1.37 hours while in the studies in which initial plasma cortisol levels were suppressed below $50 \mu \mathrm{g} / \mathrm{l}$ from the previous dexamethasone dose, mean dexamethasone half life was $4 \cdot 57 \pm 3 \cdot 20$ hours, a statistically significant difference $(t=$ 2.6666; $\mathrm{p}<0.02)$.

(4) the mean times to achieve minimum suppressed plasma cortisol levels were similar $(t=0.5543$, $\mathrm{p}<0.60$ ) in these same 10 studies (mean 6.0 \pm SD 1.85 hours), and in the studies in which initial cortisol suppression was present (mean 5.67 \pm SD 2.07 hours). However, minimum cortisol levels were statistically significantly higher $(t=3 \cdot 2866 ; p<$ 0.005 ) in these 10 studies (mean $21.1 \pm$ SD 13.8 $\mu \mathrm{g} / \mathrm{l})$ than in the studies in which initial cortisol suppression had been present (mean 10.5 \pm SD 4.9 $\mu \mathrm{g} / \mathrm{l})$.

Thus there appeared to be a correlation between shortened dexamethasone half lives and less complete suppression of cortisol production, both from the dexamethasone dose under study and from the previous dexamethasone dose.

\section{Discussion}

Not unexpectedly, the present study showed that the high dexamethasone doses often used in neurological and neurosurgical practice were capable of suppressing endogenous cortisol production and usually of maintaining the suppression for at least 6 to 8 hours in all 40 instances in which the phenomenon was studied. In the two subjects in whom dexamethasone was given in a single large bolus dose each 48 hours the investigation showed that cortisol suppression to some extent passed off between dexamethasone doses. This, of course, reflects in part the rationale for administering steroids in high dosage at rather long intervals. However, what was perhaps unexpected was that, in the 35 studies not carried out either at the time of the first dexamethasone dose or in the two persons receiving second daily bolus steroid therapy, there were 10 in which plasma cortisol levels were above the suppression criterion at the outset of study (some 8 to 12 hours after the most recent dexamethasone dose). Of these 10 studies, in six plasma cortisol values were within the physiological range for the variable (100-250 $\mu \mathrm{g} / \mathrm{l})$, though the values may still have been suppressed to some extent in relation to the affected individual's normal levels for the time of day. This relative failure to maintain suppressed cortisol production for as long as anticipated, in persons shown capable of such suppression in the present study, occurred with daily dexamethasone doses often 5 to 10 times greater than those (1-2 mg per day) known to suppress cortisol production for at least 8 hours in normal subjects. The key to this relatively incomplete suppression in some subjects may lie in the more rapid elimination of dexamethasone in the group whose cortisol production was less suppressed both from the dexamethasone dose under study, and from the previous dose. More than a decade ago Jubiz et al. ${ }^{\circ}$ showed that patients treated with phenytoin (resulting in a shortened dexamethasone half life) failed to achieve cortisol suppression with $2 \mathrm{mg}$ dexamethasone daily doses, but did achieve suppression with doses of $8 \mathrm{mg}$ daily. Meikle ${ }^{7}$ has demonstrated the importance of achieving a sufficient plasma dexamethasone level before concluding that adrenal cortical function cannot be suppressed in a given individual. The present investigation has also shown the phenomenon of decreased suppression in relation to shortened dexamethasone half-lives, though in this study even higher daily dexamethasone doses than those used by Jubiz et $a l^{\circ}$ did not invariably suppress adrenal cortical function. Five of the present subjects who failed to show full suppression were, like the cases of Jubiz et al, ${ }^{6}$ also taking phenytoin which increases 
dexamethasone clearance. From the available data it was impossible to determine whether the lessened suppression of adrenal cortical function after each steroid dose in certain subjects was solely a result of a shorter presence in the body of dexamethasone, or whether an element of non-pharmacokinetic tolerance to dexamethasone was also involved.

The findings of the present study, showing that the high dexamethasone dosage regimens sometimes used in neurology and neurosurgery do not inevitably cause profound and continued adrenal cortical suppression for as long as might have been expected, may help explain why cessation of such therapy does not more often lead to adrenal crisis. Such adrenal insufficiency seems to occur in practice very much less frequently than might be expected from knowledge of the behaviour of the adrenal cortex in the dexamethasone suppression test. The finding that high dose dexamethasone can be given each 6 or 8 hours, (though perhaps witlì a dosage gap of up to 12 hours overnight), without necessarily causing continuous adrenal suppression also raises the possibility that spacing high dosage dexamethasone intake to an 8 to 12 hourly basis, or ensuring one 10-12 hour period without dosage each day, preferably overnight, could further reduce the risk of subsequent adrenal insufficiency (though of course it would be essential to ensure that therapeutic benefit from the dexamethasone was not impaired by the wider dosage spacing). It thus seems possible that there may be room for further refining of high dosage dexamethasone regimens in neurology and neurosurgery, while maintaining the efficacy and enhancing the safety of the therapy.

\section{References}

' Green HS, Kane JM. The dexamethasone suppression test in depression. Clin Neuropharmacol 1983;6:7-24.

2 Peck CC, Barrett BB. Nonlinear least squares regression programs for microcomputers. J Pharmacokinet Biopharm 1979; 7:537-41.

${ }^{3}$ Horwitz DL, Homer LD. Analysis of Biomedical Data by Time-sharing Computers. 1. Non-linear Regression Analysis. Project No. MR005. 20-0287. Naval Medical Research Institute. National Naval Medical Centre, Bethesda, Maryland, 20014, USA. 1970.

${ }^{4}$ Marquardt, DM. An algorithm for least-squares estimation of nonlinear parameters. J Society for Industrial and Applied Mathematics. 1963,11:431-41.

${ }^{5}$ Cham BE, Sadowski B, O'Hagan JM, DeWytt CM, Bochner F. Eadie MJ. High performance liquid chromatographic assay of dexamethasone in plasma and tissue. Therap Drug Monit 1980;2:373-7.

- Jubiz W, Meikle AW, Levinson RA, Mizutani S, West $\mathrm{CD}$, Tyler FH. Effect of diphenylhydantoin on the metabolism of dexamethasone. Mechanism of the abnormal dexamethasone suppression in humans. New Engl J Med 1970;283:11-4.

${ }^{7}$ Meikle AW. Dexamethasone suppression tests: usefulness of simultaneous measurement of plasma cortisol and dexamethasone. Clin Endocrinol 1982;16:401-8 